\section{AB0709 1 SERUM IL-12/23 AND IL-17 LEVELS IN PATIENTS WITH SPONDYLOARTHRITIS WERE NOT INFLUENCED BY TNF-BLOCKADE}

M. Murakami ${ }^{1,2}$, N. Nishimoto ${ }^{1,2} .{ }^{1}$ Osaka Rheumatology Clinic, Osaka, Japan; ${ }^{2}$ Tokyo Medical University, Osaka, Japan

Background: Spondyloarthritis $(\mathrm{SpA})$ refers to a heterogeneous group of disorders with clinical features that can include axial and peripheral arthritis, inflammatory bowel disease, uveitis, and psoriasis. Several cytokines including interleukin (IL)-12/23, IL-17 and tumor necrosis factor (TNF) are involved in pathogenesis of SpA. It is assumed that TNF is the upstream cytokine in the cytokine cascade (Schett, et al., 2013).

Objectives: To investigate whether TNF inhibitors decrease serum IL-12/23 and IL-17 levels in patients with SpA.

Methods: Serum were obtained from $23 \mathrm{SpA}$ patients (AS, 10 patients; PsA, 13 patients) enrolled in this study, at baseline, 24 and 48 weeks of TNF inhibitor treatment. Serum IL-12/23 and IL17 levels were measured using LEGEND MAX Human IL-12/IL-23 (p40) ELISA Kit (BioLegend) and Human IL-17A ELISA kit (Invitrogen), respectively. IL-6 levels, the other downstream cytokine, was measured using Lumipulse G600II (FUJIREBIO) as a control.

Results: Any significant reduction in IL-12/23 levels $(143.9 \pm 143.6 \mathrm{pg} / \mathrm{mL}$ at baseline, $156.3 \pm 117.1 \mathrm{pg} / \mathrm{mL}$ at 24 weeks and $139.3 \pm 118.1 \mathrm{pg} / \mathrm{mL}$ at 48 weeks), as well as that in IL-17 levels $(13.6 \pm 51.9 \mathrm{pg} / \mathrm{mL}$ at baseline, $12.3 \pm 41.4 \mathrm{pg} / \mathrm{mL}$ at 24 weeks and $11.6 \pm 39.2 \mathrm{pg} / \mathrm{mL}$ at 48 weeks) were not observed in $23 \mathrm{SpA}$ patients. On the other hand, serum IL- 6 levels were significantly decreased after treatment $(4.0 \pm 4.2 \mathrm{pg} / \mathrm{mL}$ at baseline; $1.8 \pm 1.4 \mathrm{pg} / \mathrm{mL}, p=0.002$, at 24 weeks; $1.6 \pm 1.9 \mathrm{pg} / \mathrm{mL}$, $p=0.0002$ at 48 weeks. Pain-VAS was significantly reduced at 24 and 48 weeks compared with that at baseline. No significant differences in serum levels of analyzed cytokines were observed in the AS group compared with the PsA group at baseline (IL-12/23 levels: $110.8 \pm 70.0$ vs. $169.3 \pm 180.3 \mathrm{pg} / \mathrm{mL}, p=0.28$; IL-17 levels: $26.8 \pm 78.6$ vs. $3.4 \pm 7.6 \mathrm{pg} / \mathrm{mL}, p=0.08)$.

Conclusion: TNF inhibitors did not alter serum IL-12/23 and IL-17 levels but reduced IL-6 levels in patients with SpA. These results imply that IL-12/23 and IL-17 expression might be regulated by alternative pathways.

References:

[1] Schett, et al. Toward a cytokine-based disease taxonomy. Nat Med. 2013 Jul; 19 (7):822-4

Disclosure of Interests: Miho Murakami Grant/research support from: Chugai, Eisai, Consultant of: Chugai, Norihiro Nishimoto Grant/research support from: Chugai, Eisai, Consultant of: Chugai, Speakers bureau: Chugai, Eisai, AYUMI, Mitsubishi Tanabe, AbbVie, Novartis, Nippon Kayaku, TOWA, Astellas, Kyowa Kirin

DOI: 10.1136/annrheumdis-2020-eular.5624

\begin{tabular}{|l}
\hline AB0710 \\
PATIENT PERCEPTIONS OF FIBROMYALGIA \\
SYMPTOMS AND THE OVERLAP WITH AXIAL \\
SPONDYLOARTHRITIS
\end{tabular}

K. Gavigan ${ }^{1}$, W. B. Nowell ${ }^{1}$, R. Reynolds ${ }^{1}$, L. Stradford ${ }^{1}$, J. Curtis ${ }^{2}$, A. Ogdie ${ }^{3}$. ${ }^{1}$ Global Healthy Living Foundation, Upper Nyack, United States of America;

${ }^{2}$ University of Alabama at Birmingham, Birmingham, United States of America; ${ }^{3}$ University of Pennsylvania, Philadelphia, United States of America

Background: In clinical practice, it is often challenging to distinguish fibromyalgia syndrome (FMS) from axial spondyloarthritis (axSpA), which includes ankylosing spondylitis and non-radiographic axSpA. ${ }^{1,2}$ Early stages of axSpA may present with an onset similar to $\mathrm{FMS},{ }^{3}$ and likewise patients with FMS may have symptoms that are similar to axSpA. Differentiating between axSpA and FMS can also be challenging for patients and cause confusion about their diagnosis. Objectives: To examine the prevalence of axSpA symptoms among patients with FMS and differences in the pathway to diagnosis among patients with and without concomitant axSpA.

Methods: Adult US patients with FMS without concomitant rheumatoid arthritis or psoriatic arthritis in the ArthritisPower registry received email invitations to participate. Participants (pts) were asked whether they had a diagnosis of axSpA or ankylosing spondylitis and completed patient-reported outcome measures including Patient Reported Outcomes Measurement Information System (PROMIS) measures for Pain Interference, Sleep Disturbance and Fatigue, and the Bath Ankylosing Spondylitis Disease Activity Index (BASDAI). Pts then responded to a 57-item customized survey developed by the researchers in collaboration with patient partners. Results are descriptively reported.

Results: As of January 2020, 231 pts completed the survey; $97 \%$ female, $89 \%$ White, mean (SD) age of 52 (11). Mean (SD) Pain Interference score was 68 (5); Sleep Disturbance 63 (8); Fatigue 68 (7); and BASDAI 46 (9). Of the pts, 40 (17\%) reported concomitant axSpA, $64 \%$ osteoarthritis, $6 \%$ gout, $5 \%$ Crohn's or ulcerative colitis, and $4 \%$ lupus. Half of all pts perceived their FMS to be 'rarely' or 'never' well managed and $80 \%$ felt that they have had an undiagnosed condition in addition to their FMS and their other current diagnoses. Three-fourths
(75\%) of pts reported being able to tell the difference between their FMS pain and pain they experience as a part of the concomitant disorder. Back pain lasting $>3$ months was reported by $95 \%$ of axSpA pts and $94 \%$ of non-axSpA pts and $12 \%$ reported all of the symptoms consistent with patient reported versions of the Assessment of SpondyloArthritis International Society (ASAS) criteria (back/ buttock pain $>3$ months; age of symptom onset $<45$; sacroilitis diagnosis; at least on spondyloarthritis feature) (Figure 1), and of these, 39\% reported an axSpA diagnosis. More pts with axSpA received their FMS diagnosis by a rheumatologist $(45 \%)$ than without (41\%) (Figure 2), and of the pts without an axSpA diagnosis $(n=191)$, only $6 \%$ had recalled their provider ever discussing with them the possibility of axSpA, including non-radiographic axSpA diagnosis. Half $(53 \%)$ of pts with axSpA believe that their axSpA should have been diagnosed earlier, with $33 \%$ reporting that one reason for the delay was their doctors' belief that FMS was the cause of any axSpA symptoms they experienced.

Conclusion: Patients with FMS often experience symptoms of axSpA and the two conditions can occur concomitantly. Additional research is needed to improve the triage, diagnosis, and education of patients with FMS and symptoms of axSpA.

References:

[1] Roussou E, et al. Clin Ex Rheum Suppl. 2012;30(74):24-30.

[2] Kaskari D, et al. Mod Rheum. 2017;27(5):875-880.

[3] Hauser W, et al. Pain Rep. 2017;2(3):e598.
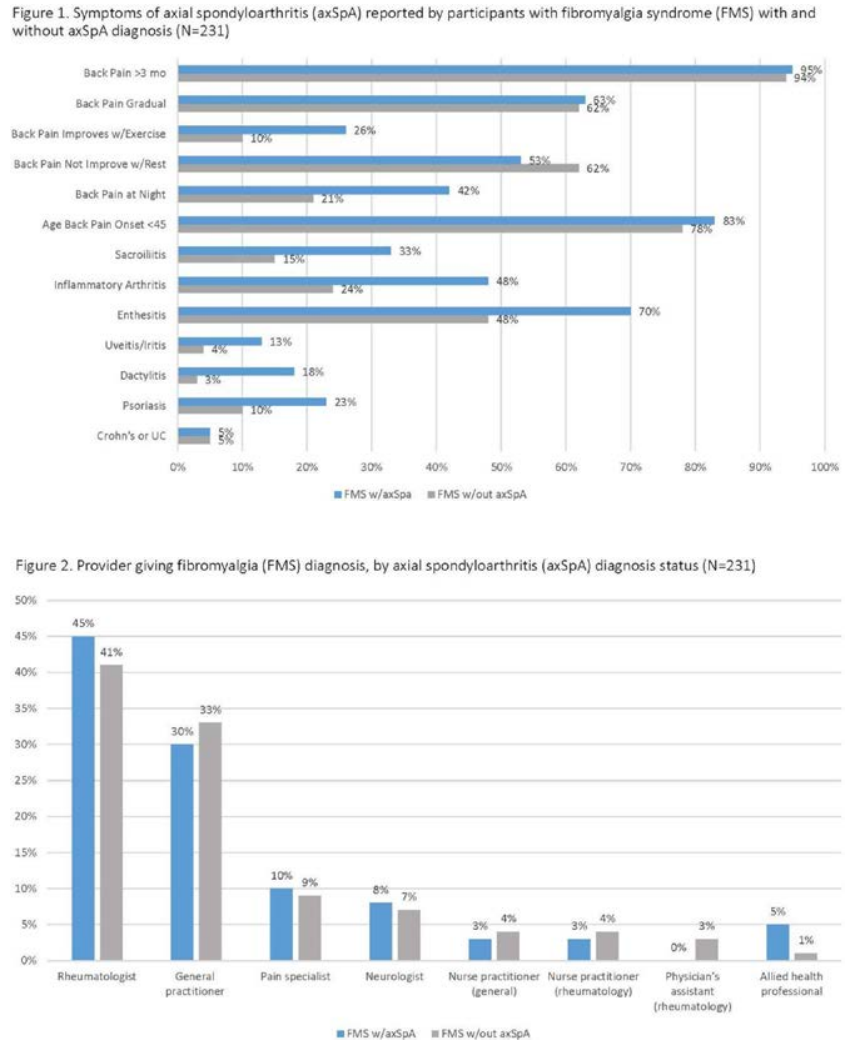

Disclosure of Interests: Kelly Gavigan: None declared, W. Benjamin Nowell: None declared, Regan Reynolds: None declared, Laura Stradford: None declared, Jeffrey Curtis Grant/research support from: AbbVie, Amgen, Bristol-Myers Squibb, Corrona, Janssen, Lilly, Myriad, Pfizer, Regeneron, Roche, UCB, Consultant of: AbbVie, Amgen, Bristol-Myers Squibb, Corrona, Janssen, Lilly, Myriad, Pfizer, Regeneron, Roche, UCB, Alexis Ogdie Grant/research support from: Pfizer, Novartis, Consultant of: Abbvie, Amgen, BMS, Celgene, Corrona, Janssen, Lilly, Pfizer, Novartis

DOI: 10.1136/annrheumdis-2020-eular.2356

\section{$\mathrm{AB} 0711$ \\ USEFULNESS OF THE TRABECULAR BONE SCORE AS A PREDICTOR OF VERTEBRAL FRACTURE IN PATIENTS WITH AXIAL SPONDYLOARTHROPATHY}

A. V. Orenes Vera ${ }^{1}$, L. Montolio-Chiva ${ }^{1}$, I. Vázquez-Gómez' ${ }^{1}$, E. Flores', E. Valls-Pascual', À. Martínez-Ferrer ${ }^{1}$, D. Ybañez ${ }^{1}$, L. García-Ferrer ${ }^{1}$, M. VegaMartínez ${ }^{2}$, M. Graells-Ferrer ${ }^{2}$, A. Sendra-García ${ }^{1}$, V. Núñez-Monje ${ }^{1}$, I. Torner Hernández ${ }^{1}$, J. J. Alegre-Sancho ${ }^{1} .{ }^{1}$ Hospital Doctor Peset, Valencia, Spain; ${ }^{2}$ Hospital Doctor Peset, Radiodiagnóstico, Valencia, Spain 
Background: In axial spondyloarthritis (axSpA) the risk of vertebral fracture is increased, not always corresponding with the values of bone mineral density (BMD). One possible explanation is that syndesmophytes interfere with these values. We consider whether the evaluation of trabecular microarchitecture by an accessible methodlike the Trabecular Bone Score (TBS), that does not involve additional irradiation neither seem to be influenced by the presence of syndesmophytes, may be an advantage to estimate the risk of fracture.

Objectives: To estimate the prevalence of vertebral fractures in patients with axSpA. To assess the diagnostic accuracy of TBS and BMD for vertebral fracture, and if it is influenced by the presence of syndesmophytes. To analyze the correlation between the absolute values of BMD and TBS in the lumbar spine.

Methods: Cross-sectional study. Patients were consecutive recruited. We collected demographic (sex, age), clinical (syndesmophytes, vertebral fracture, BASDAI, BASFI, time of evolution of axSpA, treatment) and analytical variables [vitamin D (1,25-OHD), CRP and ESR]. The BMD was determined using the Lunar Prodigy ProTM densitometer from GE Healthcare, to which the TBS iNsight $₫$ software version 2.2 was added to perform the TBS analysis. The presence of fracture was evaluated by radiology. The statistical analysis was performed with the SPSS 22.0 and OpenEpi softwares.

Results: 84 patients were included, 60 men and 24 women, with a mean age of 59 years ( \pm SD 13). $51.2 \%$ had lumbar syndesmophytes. The prevalence of fractures was $13.7 \%, 95 \mathrm{Cl}$ (7.8-22.9). 51.2\% were treated with NSAIDs, and $48.8 \%$ with biological drugs. The evolution of axSpA was $>10$ years in $65.5 \%$. The mean scores of BASDAI and BASFI were 3.7 and 4.3 respectively ( \pm SD 2.2 and 2.3 ). The mean CRP value was $8.5 \mathrm{mg} / \mathrm{L}( \pm \mathrm{SD} 8.4)$, ESR $12.2 \mathrm{~mm} / \mathrm{h}( \pm \mathrm{SD} 11.4)$ and 1.25-OHD 27.9ng / dL ( \pm SD 13.6).

According to the lumbar and femoral T Score, $9.5 \%$ and $15.5 \%$ of the patients were in the range of osteoporosis respectively. $19 \%$ patients had a low TBS value ( $\leq 1.23)$.

Regarding the influence of syndesmophytes on TBS and BMD values, we found significant differences in lumbar spine BMD $(p=0.01)$ but not in total hip and femoral neck BMD ( $p=0.2$ and 0.3 respectively) nor in the TBS $(p=0.1)$.

Regarding the correlation of TBS and BMD values of the spine, no correlation was observed in patients with syndesmophytes, while a moderate correlation $(r=0.4, p=0.02$ ) was observed in patients without syndesmophytes.

In the univariate analysis, the factors related to the presence of vertebral fracture were age, female sex, absolute BMD values in the lumbar spine and total hip, and TBS values. No relationship was found with the rest of the variables. In the multivariate analysis, only the TBS showed a significant association with the presence of fractures $(p=0.02)$.

Regarding the predictive capacity of fractures, TBS showed a higher sensitivity than that of BMD $(55.6 \%$ versus $18.2 \%$ and $30 \%$ of BMD in the spine and hip respectively), being the specificity comparable $(85.3 \%$ versus $91.3 \%$ and $85.1 \%$ of BMD in column and hip respectively).

Conclusion: the prevalence of fractures was $13.7 \%$ among the patients studied, $95 \mathrm{Cl}$ (7.8-22.9). The presence of syndesmophytes influenced the values of lumbar BMD but not the hip BMD or those of the TBS. We found a correlation between the values of BMD of the spine and TBS only in patients who did not have syndesmophytes. Only TBS values were significantly related to the presence of fractures in the multivariate analysis. TBS showed greater sensitivity with similar specificity than BMD for the detection of vertebral fractures.

Disclosure of Interests: Ana V Orenes Vera: None declared, L Montolio-Chiva: None declared, I Vázquez-Gómez: None declared, Eduardo Flores: None declared, Elia Valls-Pascual Grant/research support from: Roche, Novartis, and AbbVie, Speakers bureau: AbbVie, Lilly, Pfizer, MSD, Novartis, Janssen, Bristol Myers Squibb, UCB Pharma, À Martínez-Ferrer: None declared, Desamparados Ybañez: None declared, Luis García-Ferrer: None declared, María Vega-Martínez: None declared, Magdalena Graells-Ferrer: None declared, A Sendra-García: None declared, V Núñez-Monje: None declared, Inmaculada Torner Hernández: None declared, Juanjo J Alegre-Sancho Consultant of: UCB, Roche, Sanofi, Boehringer, Celltrion, Paid instructor for: GSK, Speakers bureau: MSD, GSK, Lilly, Sanofi, Roche, UCB, Actelion, Pfizer, Abbvie, Novartis DOI: 10.1136/annrheumdis-2020-eular.5932

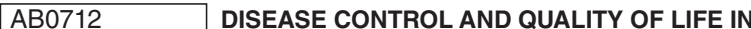 PATIENTS WITH ANKYLOSING SPONDYLITIS AND PSORIATIC ARTHRITIS IN REAL CLINICAL PRACTICE IN SPAIN: MIDAS STUDY}

J. L. Pablos ${ }^{1}$, C. Fernández-Carballido ${ }^{2}$, X. Juanola ${ }^{3}$, J. Gratacos-Masmitja ${ }^{4}$, E. De Miguel ${ }^{5}$, R. Ariza-Ariza ${ }^{6}$, C. Sanabra ${ }^{7}$, P. Terradas ${ }^{7}$, C. Sastré ${ }^{7}{ }^{1} \mathrm{H}_{\text {ospital }}$ 12 de Octubre, Madrid, Spain; ${ }^{2}$ Hospital Universitario San Juan de Alicante, Alicante, Spain; ${ }^{3}$ Hospital Universitari Bellvitge, IDIBELL, L'Hospitalet de Llobregat, Barcelona, Spain; ${ }^{4}$ Hospital Universitario Parc Taulí, Sabadell, Barcelona, Spain; ${ }^{5}$ Hospital Universitario La Paz, Madrid, Spain; ${ }^{6}$ Hospital
Universitario Virgen de Macarena, Sevilla, Spain; ${ }^{7}$ Novartis Farmacéutica, Barcelona, Spain

Background: There are few data on disease activity status and Quality of Life (QoL) in clinical practice in Spain for patients with ankylosing spondylitis (AS) and psoriatic arthritis (PsA).

Objectives: To assess the disease activity status in standard clinical practice through BASDAl $<4$ in AS and DAPSA $\leq 14$ in PsA and the relationship with QoL.

Methods: An observational, non-interventional, cross-sectional, multicenter study. Patients $\geq 18$ years with $\geq 6$ months diagnosis and classified by ASAS and modified New York criteria or CASPAR criteria undergoing treatment $\geq 3$ months. At the cross-sectional visit, the principal variable taken was the percentage of patients under remission and low disease activity according to the national and European recommendations1-3, assessed through BASDAI and ASDAS-CRP in AS or DAPSA and MDA in PSA. The relationship between patients' QoL and dis ease activity were assessed using ASAS-HI in AS and PSAID in PSA.

Results: 313 AS patients were included, $75.7 \%$ male, $78.5 \%$ HLA-B ${ }^{\star} 27+$, with a mean (SD) age of 50.4 (12.0) years, a mean (SD) disease duration of 15.5 (11.6) years and a mean (SD) CRP of 5.1 (8.2) mg/l. 313 PsA patients were included, $54.3 \%$ male, $17.95 \%$ HLA-B*27+, with a mean (SD) age of 54.1 (12.2) years, a mean (SD) disease duration of 10.5 (9.0) years and a mean (SD) CRP of 4.91 (7.3) $\mathrm{mg} / \mathrm{l}$. $29.7 \%$ AS patients were on biological and $26.8 \%$ were on non-biological therapy vs $17.9 \%$ and $40.9 \%$ PsA patients, respectively. According to BASDAI, 64.5\% AS patients were on LDA and 29.4\% PsA patients had inactive disease by ASDAS-CRP, $59.4 \%$ of patients had a DAPSA $<14$ while $19,8 \%$ were on remission also by DAPSA. In both groups, the QoL impact was low, mean 5.8 in ASAS-HI by AS and 3.0 by PSAID in PSA. QoL impact was significantly higher in patients with active disease (9.3 in AS and 4.4 in PsA).

Conclusion: Our observations show that most AS and PsA patients have an inactive disease, whereas $36 \%$ and $41 \%$ of AS and PsA patients, respectively, are inadequately controlled despite therapy in standard clinical practice in Spain, which is associated to a significantly worse QoL.

References:

[1] Torre Alonso JC et al. Reumatol Clin 2018;14:254-68

[2] Smolen JS et al. Ann Rheum Dis 2018;77:3-17

[3] Gratacós J et al. Reumatol Clin 2018;14:320-33

Table 1. AS and PsA scores according to the current treatment.

\begin{tabular}{|c|c|c|c|}
\hline & Valid $N$ & Mean (SD) & $95 \%(\mathrm{Cl})$ \\
\hline \multicolumn{4}{|l|}{ AS } \\
\hline \multicolumn{4}{|l|}{ Disease control } \\
\hline \multicolumn{4}{|l|}{ (BASDAI) } \\
\hline Not under remission & 111 & $9.3(3.7)$ & $(8.6 ; 10.0)$ \\
\hline \multicolumn{4}{|l|}{ (BASDAI<4) } \\
\hline Under remission & 202 & $3.9(3.4)$ & $(3.4 ; 4.4)$ \\
\hline \multicolumn{4}{|l|}{ (BASDAl $\geq 4)$} \\
\hline Total & 313 & $5.8(4.4)$ & $(5.3 ; 6.3)$ \\
\hline \multicolumn{4}{|l|}{ Disease activity } \\
\hline \multicolumn{4}{|l|}{ (ASDAS-CRP) } \\
\hline ASDAS-CRP $\geq 1.3$ & 221 & $7.1(4.1)$ & $(6.6 ; 7.7)$ \\
\hline ASDAS-CRP $<1.3$ & 92 & $2.7(3.4)$ & $(2.0 ; 3.4)$ \\
\hline Total & 313 & $5.8(4.4)$ & $(5.3 ; 6.3)$ \\
\hline \multicolumn{4}{|l|}{ PsA } \\
\hline \multicolumn{4}{|l|}{ Disease control } \\
\hline \multicolumn{4}{|l|}{ (DAPSA) } \\
\hline Not under remission & 127 & $4.5(2.4)$ & $(4.1 ; 4.9)$ \\
\hline \multicolumn{4}{|l|}{ (DAPSA>14) } \\
\hline Under remission & 186 & $1.9(1.7)$ & $(1.7 ; 2.2)$ \\
\hline \multicolumn{4}{|l|}{ (DAPSA $\leq 14)$} \\
\hline Total & 313 & $3.0(2.4)$ & $(2.7 ; 3.2)$ \\
\hline \multicolumn{4}{|l|}{ Active disease (MDA) } \\
\hline Inactive & 161 & $1.5(1.4)$ & $(1.3 ; 1.7)$ \\
\hline \multicolumn{4}{|l|}{ (MDA criteria $\geq 5$ ) } \\
\hline Active & 152 & $4.5(2.2)$ & $(4.2 ; 4.8)$ \\
\hline \multicolumn{4}{|l|}{ (does not meet MDA criteria) } \\
\hline Total & 313 & $3.0(2.4)$ & $(2.7 ; 3.2)$ \\
\hline
\end{tabular}

\section{Acknowledgments: MIDAS group}

Disclosure of Interests: José L. Pablos Consultant of: Pfizer, Lilly, Novartis, Roche, Celgene, Sanofi, Gilead, Biogen, Paid instructor for: Bristol, Speak ers bureau: Abbvie, Janssen, Pfizer, Lilly, Novartis, Roche, Celgene, Bristol Sanofi, Cristina Fernández-Carballido Consultant of: Yes, I have received fees for scientific advice (Abbvie, Celgene, Janssen, Lilly and Novartis) Speakers bureau: Yes, I have received fees as a speaker (Abbvie, Celgene, Janssen, Lilly, MSD, Novartis), Xavier Juanola Consultant of: Pfizer Lilly, Novartis, Roche, Celgene, Sanofi, Gilead, Biogen, Paid instructor for: Bristol, Speakers bureau: Abbvie, Janssen, Pfizer, Lilly, Novartis, Roche, Celgene, Bristol, Sanofi, Jordi Gratacos-Masmitja Grant/research support from: a grant from Pfizzer to study implementation of multidisciplinary units 